\title{
Persistently increased systemic, but not cardiac-specific, adhesion molecule expression and coronary endothelial dysfunction in human cardiac allografts
}

\author{
Stephen M. Wildhirt, MD, PhD, ${ }^{\text {a }}$ Costas Schulze, MD, ${ }^{\text {b }}$ Nicole Conrad, PhD, ${ }^{\text {b }}$ Robert Bauernschmitt, MD, \\ Rüdiger Lange, $M D^{,}{ }^{a}$ and Wolfgang von Scheidt, $M^{c}$
}

\section{eonly Additional material is available online.}

Background: Adhesion molecules are involved in inflammatory processes that alter endothelial function and lead to impairment of coronary vasomotor function. We studied a possible relationship between systemic expression, cardiac-specific expression, or both of P-selectin and intercellular adhesion molecule 1 and coronary vasomotor function both 1 and 12 months after heart transplantation in human subjects.

Methods: The expression of endomyocardial and soluble forms of P-selectin and intercellular adhesion molecule 1 , as well as levels of tumor necrosis factor $\alpha$, were determined in aortic and coronary sinus blood samples 1, 6, and 12 months after heart transplantation in 42 transplant recipients and 20 age-matched, nontransplanted control subjects. In addition, both endothelium-dependent (acetylcholine) and endothelium-independent (adenosine) coronary vasomotor function were assessed by using a Doppler flow wire and quantitative coronary angiography 1 and 12 months after heart transplantation.

Results: Adhesion molecules were highly expressed 1 month after heart transplantation and remained at high levels 12 months after heart transplantation when compared with levels in nontransplanted control subjects. No cardiac-specific expression or release of P-selectin or intercellular adhesion molecule 1 was observed. There was a significant inverse correlation between coronary vasomotor function and soluble adhesion molecule expression both 1 and 12 months after heart transplantation.

Conclusion: Persistently high levels of circulating adhesion molecules are of systemic, but not cardiac-specific, origin and reflect a chronic inflammatory state throughout the first year after heart transplantation. This is associated with impairment of coronary vasomotor function, an early and potentially reversible step in the process of atherothrombosis and transplant coronary artery disease.

visions received May 11, 2005; accepted for publication May 18, 2005

Address for reprints: Stephen M. Wildhirt, $\mathrm{MD}, \mathrm{PhD}$, Department of Cardiovascular Surgery, German Heart Center Munich, Technical University of Munich, Lazarettstr. 46 of Munich, Lazarettstr. 36, 80636 Munich, Germany (E-mail: wildhirt@gmx.net).

J Thorac Cardiovasc Surg 2005;130:1175-82 0022-5223/\$30.00

Copyright (C) 2005 by The American Association for Thoracic Surgery

doi:10.1016/j.jtcvs.2005.05.053
$\mathrm{S}$ oluble adhesion molecules have been shown to be markers and mediators of endothelial dysfunction in various disease states, including arteriosclerosis, myocardial infarction, and heart failure. ${ }^{1}$ It has been shown that early expression of both P-selectin and intercellular adhesion molecule 1 (ICAM-1) is predictive of future cardiovascular events in apparently healthy men and in patients with documented coronary artery disease., ${ }^{2,3}$

Cardiac transplantation has been the established treatment of choice for end-stage heart failure of various causes. However, the limiting factor for long-term survival 


$$
\begin{aligned}
& \text { Abbreviations and Acronyms } \\
& \begin{array}{ll}
\text { Ach } & =\text { acetylcholine } \\
\text { CFVR } & =\text { coronary flow velocity reserve } \\
\text { HTx } & =\text { heart transplantation } \\
\text { ICAM-1 } & =\text { intracellular adhesion molecule } 1 \\
\text { IVUS } & =\text { intravascular ultrasound } \\
\text { Co } & =\text { control } \\
\text { LAD } & =\text { left anterior descending coronary artery } \\
\text { TNF- } \alpha & =\text { tumor necrosis factor } \alpha
\end{array}
\end{aligned}
$$

is the progressive development of transplant coronary artery disease. The disease process leads to vanishing of small intramyocardial resistance vessels but also involves epicardial conduit vessels. Its nature is multifactorial, consisting of immunologic and classic cardiovascular risk factors. ${ }^{4}$ One early predictor of transplant coronary artery disease is the development of endothelial dysfunction in response to endothelium-dependent and endothelium-independent vasodilators. ${ }^{5,6}$

Increased circulating adhesion molecule levels serve as markers and mediators for future cardiovascular events. In addition, it has been shown that during chronic rejection, P-selectin expression correlated with the intensity of arterial intimal thickening. ${ }^{7}$ However, it remains unclear whether soluble adhesion molecules are of systemic or cardiacspecific origin. Moreover, a direct association between circulating adhesion molecules and coronary vasomotor dysfunction has not been shown. This prompted us to investigate the potential role of systemic expression, cardiacspecific expression, or both of soluble P-selectin and ICAM-1 in the development of coronary endothelial dysfunction in human heart transplant recipients.

\section{Material and Methods}

A total of 62 patients, including 42 heart transplant recipients and 20 age-matched control subjects, undergoing coronary artery bypass grafting were included in this prospective and longitudinally designed study. The demographics are given in Table 1 .

In transplant recipients triple-drug immunosuppression based on tacrolimus or cyclosporine (INN: ciclosporin) in combination with myocophenolate was initiated immediately after organ implantation and was maintained throughout the study. Other medications, including angiotensin-converting enzyme inhibitors, calcium antagonists, statins, and diuretics, were discontinued 24 to 48 hours before each examination. All patients agreed to participate in the study and provided written informed consent. The study protocol was approved by the Ethics Committee at the University of Munich, Germany. No study-related complications and no deaths occurred during the 1-year follow-up.

Blood samples from 20 age-matched, nontransplanted control subjects undergoing coronary bypass surgery with the use of cardiopulmonary bypass were collected at similar time points to account for differences in adhesion molecule and cytokine expres- sion. This was done to distinguish between the effects of the cardiac surgical procedure with cardiopulmonary bypass per se and the effects of the alloimmune response under immunosuppresive therapy (demographics are shown in Table 1).

\section{Study Protocol}

Left heart catheterization was performed at 1 and 12 months after heart transplantation (HTx) and consisted of a measurement of hemodynamics and routine coronary angiography to exclude donor-transmitted coronary artery disease. In addition, endotheliumdependent and endothelium-independent epicardial (quantitative coronary angiography) and microvascular (Doppler flow wire) coronary vasomotor function were assessed.

Blood samples were drawn from the peripheral vein, coronary sinus, and aorta. Endomyocardial biopsy specimens were obtained from the interventricular septum for determination of ICAM and P-selectin protein expression at months 1, 6, and 12 after transplantation. To account for differences in sample quality, we always used tissues from 2 biopsy samples for immunohistochemistry.

\section{Functional Assessment of Coronary Vasomotor Function}

Measurement of coronary vasomotor response (assessed by means of quantitative coronary angiography and intracoronary Doppler flow measurement) and intimal thickening has been described previously. $^{8}$

In brief, after administration of 5000 units of heparin, a Doppler flow wire (0.018 in [0.04 cm]; Flowire, Cardiometrics Inc) was placed in the proximal left anterior descending coronary artery (LAD) to assess coronary blood flow velocities continuously. After baseline flow velocity readings were obtained, adenosine (80.0 and $160.0 \mu \mathrm{g} / \mathrm{min}$ for 5 minutes each; Adrekar, Sanofi-Winthrop) was infused to assess maximal endothelium-independent coronary flow. Thereafter, epicardial and microvascular endothelial coronary vasomotor function was determined with intracoronary administration of acetylcholine (Ach; 1.0 and $30.0 \mu \mathrm{g} / \mathrm{min}$ for 5 minutes each; estimated final concentration in the coronary bed, $10^{-7}$ to $3 \times 10^{-8} \mathrm{~mol} / \mathrm{L}$; Acetylcholine Dispersa, Germering).

At the end of each infusion, coronary arteriography was performed and analyzed off-line by using quantitative coronary angiography.

\section{Coronary Flow Reserve}

Coronary flow velocity reserve (CFVR), a marker of microvascular integrity, was expressed as the ratio of peak to baseline blood flow velocity. Heart rate, mean arterial pressure, coronary flow velocity, and electrocardiography were monitored continuously throughout the procedure. It was ensured that measurements of the flow velocity reserve of the microvascular bed were not altered by epicardial vasoconstriction. No epicardial vasoconstriction of greater than $50 \%$ during Ach infusion was observed.

An increase in flow velocity of a factor of less than 2.0 was considered pathologic, as described previously. ${ }^{9}$

\section{Quantitative Coronary Angiography}

Epicardial vasomotor response was assessed with a computerized automatic-analysis system (Hicor, Siemens). Nonstenotic proximal and distal coronary arterial segments identified between easily 
TABLE 1. Demographics of heart transplant recipients and control subjects

\begin{tabular}{|c|c|c|c|}
\hline & $\begin{array}{l}\text { HTx recipients } \\
(\mathrm{n}=42)\end{array}$ & $\begin{array}{l}\text { Control subjects } \\
(\mathrm{n}=20)\end{array}$ & $P$ value \\
\hline Study examination (days after the operation) & $37 \pm 8$ & $31 \pm 12$ & .74 \\
\hline Age (y) & $52.4 \pm 11.2$ & $53.6 \pm 9.5$ & .87 \\
\hline Weight (kg) & $72.5 \pm 12$ & $76.5 \pm 8$ & .30 \\
\hline Height $(\mathrm{cm})$ & $174 \pm 8$ & $175 \pm 10$ & .38 \\
\hline $\operatorname{Sex}(M: F), n(\%)$ male & $38(82)$ & $17(93)$ & .12 \\
\hline Number of CAD risk factors per patient & 1.31 & 1.36 & .67 \\
\hline \multicolumn{4}{|l|}{ Native CAD risk factors } \\
\hline Diabetes, n (\%) & $6(14)$ & $8(12)$ & .67 \\
\hline Hypertension, n (\%) & $8(19)$ & $10(19)$ & .58 \\
\hline Hyperlipidemia, n (\%) & $21(50)$ & $7(51)$ & .66 \\
\hline Tobacco, n (\%) & $16(38)$ & $11(55)$ & .06 \\
\hline Hyperuremia, n (\%) & $5(12)$ & $2(10)$ & .53 \\
\hline Alcohol, n (\%) & $3(7)$ & $2(10)$ & .57 \\
\hline Creatinine (mg/dL) & $1.3 \pm 0.4$ & $1.4 \pm 0.4$ & .77 \\
\hline Cholesterol (mg/dL) & $191 \pm 18$ & $201 \pm 21$ & .66 \\
\hline LDL (mg/dL) & $117 \pm 13$ & $112 \pm 11$ & .44 \\
\hline $\mathrm{HDL}(\mathrm{mg} / \mathrm{dL})$ & $47 \pm 8$ & $44 \pm 9$ & .71 \\
\hline $\mathrm{Lp}(\mathrm{a})(\mathrm{mg} / \mathrm{dL})$ & $24 \pm 10$ & $31 \pm 12$ & .29 \\
\hline C-reactive protein (mg/dL) & $1.6 \pm 0.5$ & $2.1 \pm 0.6$ & .37 \\
\hline Ejection fraction (\%) & $64 \pm 9$ & $57 \pm 8$ & .62 \\
\hline MAP & $77 \pm 12$ & $75 \pm 11$ & .53 \\
\hline LVEDP & $14 \pm 7$ & $13 \pm 8$ & .71 \\
\hline ACE inhibitor (\%) & 31 & 45 & .43 \\
\hline Statins $(\%)$ & 62 & 58 & .61 \\
\hline Diuretics (\%) & 55 & 61 & .58 \\
\hline Calcium antagonists (\%) & 48 & 42 & .31 \\
\hline$\beta$-Blockers (\%) & 35 & 45 & .35 \\
\hline
\end{tabular}

Relevant demographics of recipients and nontransplanted control subjects at the time of follow-up after transplantation or coronary artery bypass grafting surgery. $C A D$, Coronary artery disease; $L D L$, low-density lipoprotein; $H D L$, high-density lipoprotein; $L p(a)$, lipoprotein $A ; M A P$, mean arterial pressure; $L V E D P$, left ventricular end-diastolic pressure; $A C E$, angiotensin-converting enzyme.

visualized branch points were selected for analysis in the LAD. Responses to the different pharmacologic stimuli were expressed as percentage changes versus baseline value and calculated as the average between the 2 segments analyzed. Intraobserver and interobserver variability showed high reproducibility $(r>0.89, P<$ .0001). Epicardial endothelial dysfunction has been described as a greater than $10 \%$ diameter reduction in response to Ach compared with baseline values. ${ }^{8}$

\section{Intravascular Ultrasonography}

After Doppler flow measurement, intravascular ultrasonography (IVUS) was performed to detect atherosclerotic changes not visible with angiography. The imaging system consisted of a $30-\mathrm{MHz}$ ultrasound transducer enclosed within an acoustic housing on the tip of a 2.9F flexible, rapid exchange catheter (CVIS Inc). The catheter was advanced to the distal LAD or left circumflex artery, carefully observing a lumen-IVUS catheter diameter ratio of greater than 1.5. During the standard pullback maneuvers, images were documented on the SVHS Videosystem for further analysis.

The 3 sites with the most prominent intimal proliferation were analyzed, and the averaged maximal intimal thickness (the greatest distance from the intimal leading edge to the media-adventitia border) was calculated.
All segments studied were classified according to both severity of intimal thickening and the degree of circumferential involvement to categorize the degree of coronary disease. The following definitions were applied: class 0 , no intimal thickening; class 1 , mild intimal thickening (intimal layer $<300 \mu \mathrm{m}$ thick involving $>180^{\circ}$ of vessel circumference); class 2 , moderate intimal thickening (intimal layer $300-500 \mu \mathrm{m}$ thick but involving $<180^{\circ}$ of vessel circumference or $>500 \mu \mathrm{m}$ thick at any point of the vessel cross-section); class 3, severe intimal thickening (intimal layer $>500 \mu \mathrm{m}$ thick but involving $>180^{\circ}$ of vessel circumference or $>1 \mathrm{~mm}$ thick at any point of the vessel cross-section).

The analysis was performed by observers without knowledge of the results of endothelial function or cytokine or adhesion molecule levels.

\section{Measurement of Tumor Necrosis Factor $\alpha$ and Soluble Adhesion Molecules}

Plasma levels were measured by using a commercially available enzyme-linked immunoabsorbant assay (TNF- $\alpha$; Medgenix GmbH; P-Selectin and ICAM-1, R\&D System Europe). Blood samples were immediately placed on ice and centrifuged. Plasma was separated, frozen in liquid nitrogen, and stored at $-80^{\circ} \mathrm{C}$ until further processing. Standards were prepared, and the appropriate 
volume of sample or standard was added to a 96-well polystyrene microtiter plate precoated with the monoclonal antibody against tumor necrosis factor $\alpha$ (TNF- $\alpha)$. All samples were run in duplicate. Each well was then aspirated, and the plates were washed with the specific surfactant provided by the manufacturer. An enzyme-linked polyclonal antibody against the cytokine was added. Substrate solution was added to each well, and the optical density was read at the appropriate wavelength for each assay.

\section{Immunohistochemical Detection of Cardiac Adhesion Molecule Expression}

Interventricular biopsy specimens were cut into sections $(3 \mu \mathrm{m}$ in thickness). At least 10 sections from each biopsy specimen were analyzed. Adjacent cryosections were used for detection of P-selectin and ICAM-1. In brief, sections were fixed with chloroform (30 minutes) and blocked with $1.5 \% \quad \mathrm{H}_{2} \mathrm{O}_{2}(10$ minutes). After washing $(2 \times 5$ minutes $)$, nonspecific binding was blocked with protein serum free block (Dako). For detection of P-selectin (dilution 1:300; Dako) and ICAM-1 (dilution 1:600; Dako), monoclonal antibodies were incubated for 30 minutes. After washing $(3 \times 5$ minutes $)$, a secondary antibody (EnVision; Dako) was applied for 30 minutes and visualized with a peroxidase substrate AEC kit (Vector Labs). After rinsing, sections were counterstained with Mayer's hemalum solution and mounted. All steps were performed at room temperature. Negative controls were obtained by omitting the primary antibody. Quantification of specific immunoreactivity was not performed because only small biopsy samples were taken from the interventricular septum. We believe that this would lead to overinterpretaion of the data obtained.

\section{Statistical Analysis}

Data are presented as mean values \pm standard deviation. Nonparametric data were analyzed by using the Mann-Whitney $U$ test, and correlations were determined with the Spearman rank test. Multiple comparisons were performed by means of analysis of variance, followed by Bonferroni post-hoc analysis. The Student $t$ test for paired and unpaired comparisons was used as appropriate. Dichotomous variables were analyzed with the $\chi^{2}$ test. The correlation coefficient was determined by means of simple regression analysis.

\section{Results}

\section{Patient Characteristics}

None of the patients included in the investigation had clinical signs of infection or acute rejection episodes of International Society for Heart and Lung Transplantation grade $1 \mathrm{~b}$ or greater during the time of sample collection and functional assessment of the coronary vasculature. Moreover, no significant differences with regard to the number of acute cellular rejection episodes were found.

The patients did not differ with regard to the number of classic atherosclerotic risk factors, such as hypertension, hyperlipdemia, diabetes, or history of smoking (Table 1).

In addition, no significant differences with regard to cardiac hemodynamics were observed at either follow-up appointment. Data are depicted in Table 1.

\section{Coronary Intimal Thickening (IVUS) and Angiographic Findings}

At 1 month after HTx, no differences concerning the extent of coronary intimal thickening were observed between patients with impaired and normal endothelium-dependent CFVR (Table E1). In addition, no evidence for coronary lesion formation was observed by means coronary angiography at 1 and 12 months after transplantation.

\section{Systemic and Cardiac-specific Adhesion Molecule Expression}

Adhesion molecules were highly expressed 1 month after HTx and remained at high levels when compared with those seen in age-matched healthy control subjects (Figure 1, A and $B$; P-selectin-Co: $34.3 \pm 5.8$; HTx: 1 month, $78.8 \pm$ 9.3; 6 months, $65.1 \pm 6.8 ; 12$ months, $47.3 \pm 2.9[P<.05$, all HTx vs Co]; ICAM-1-Co: $140.2 \pm 12.8$; HTx: 1 month, $286.7 \pm 28.3$; 6 months, $195.8 \pm 11.3$; 12 months, $192.4 \pm 10.2[P<.05])$. No significant transcardiac production or release of adhesion molecules was detected (Figure 1, $C$ and $D$ ). TNF- $\alpha$ levels remained high throughout the first year (Figure E1, $A$; Co: 1 month, $10.6 \pm 3.8 ; 6$ months, $5.8 \pm 3.8$; HTx: 1 month, $19.5 \pm 2.3 ; 6$ months, $21.5 \pm 4.8$; 12 months, $24.3 \pm 4.1[P<.05$, all HTx vs Co] $)$. A significant transcardiac release was noted and expressed as the difference between aortic and coronary sinus levels 1 year after HTx (Figure E1, B). There was no significant correlation between plasma levels of immunosuppressive drugs and adhesion molecule or cytokine expression.

Immunohistochemical analysis of endomyocardial biopsy specimens at 1 and 12 months after HTx did not reveal differences between patients with or without impairment of microvascular CVFR (Figure 2). Two biopsy samples from each time point were used; however, semiquantification of endomyocardial P-selectin and ICAM-1 immunoreactivity was not performed in this setting.

Moreover, there was a close and significant correlation between the proinflammatory cytokine TNF- $\alpha$ and adhesion molecules (Figure E2).

\section{Coronary Vasomotor Function}

Cardiac hemodynamics, including heart rate and mean blood pressure, were not altered by the administration of each drug.

Epicardial coronary diameter change after administration of Ach resulted in luminal constriction of $-5 \% \pm 2 \%$ in proximal and $-11 \% \pm 5 \%$ in distal segments at 1 month and $-7 \% \pm 3 \%$ in proximal and $-12 \% \pm 4 \%$ in distal segments at 12 months after HTx. No differences were found between patients with or without microvascular vasomotor dysfunction. Endothelium-independent epicardial vasomotor response (adenosine) was 7\% $\pm 3 \%$ in proximal segments and $17 \% \pm 6 \%$ in distal segments at 1 month, 

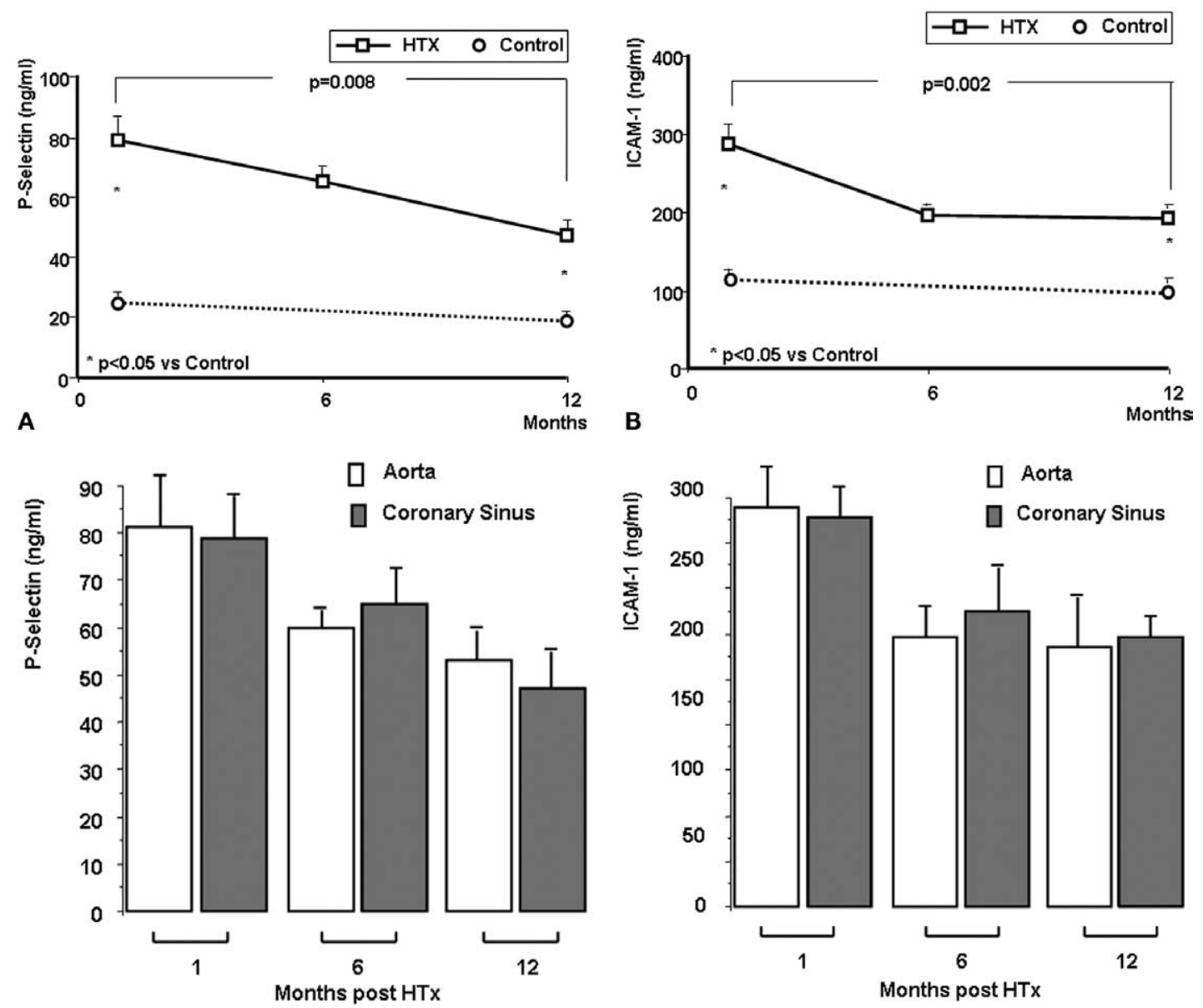

C

P-Selectin

D

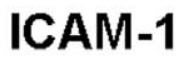

Figure 1. Adhesion molecules were highly expressed 1 month after transplantation and remained at high levels when compared with levels in age-matched healthy control subjects (A and B). No significant transcardiac production of release, expressed as the differences between aortic and coronary sinus levels of adhesion molecules, was detected (C and D). HTX, Heart transplantation; ICAM-1, intercellular adhesion molecule 1.

which is comparable with $6 \% \pm 2 \%$ in proximal and $15 \%$ $\pm 8 \%$ in distal segments seen 12 months after HTx.

Epicardial vasomotor responsiveness to both Ach and adenosine were not associated with each other.

CFVR response to intracoronary Ach 1 month after HTx was impaired $(<2.0)$ in $11(26.1 \%)$ of 42 patients. At 1 year after transplantation, $13(31 \%)$ patients presented with an impaired CFVR. There was an inverse correlation between CFVR in response to Ach and Pselectin both at 1 and 12 months after HTx (Figure 3, A and $B$ ). In addition, CFVR after intracoronary Ach was significantly associated with higher levels of ICAM-1 at 12 months after HTx (Figure 3,C). Finally, there was an inverse correlation between impaired CFVR after Ach infusion and coronary sinus TNF- $\alpha$ levels $(R=0.31, P$ $=.02)$ at 1 month after transplantation.

\section{Discussion}

The major findings of the present study are as follows. First, cardiac transplant recipients express persistently high levels of the circulating adhesion molecules P-selectin and ICAM-1 in the absence of acute rejection episodes or infection. Second, the levels of adhesion molecules appear of systemic origin because no cardiac-specific production or release was observed. Third, circulating adhesion molecule expression correlates with the severity of endotheliumdependent coronary vasomotor dysfunction early and 1 year after HTx.

\section{Origin and Specificity of Soluble Cell Adhesion \\ Molecules in Transplant Recipients}

In the present study no cardiac-specific production or release of adhesion molecules was detected, and one could 


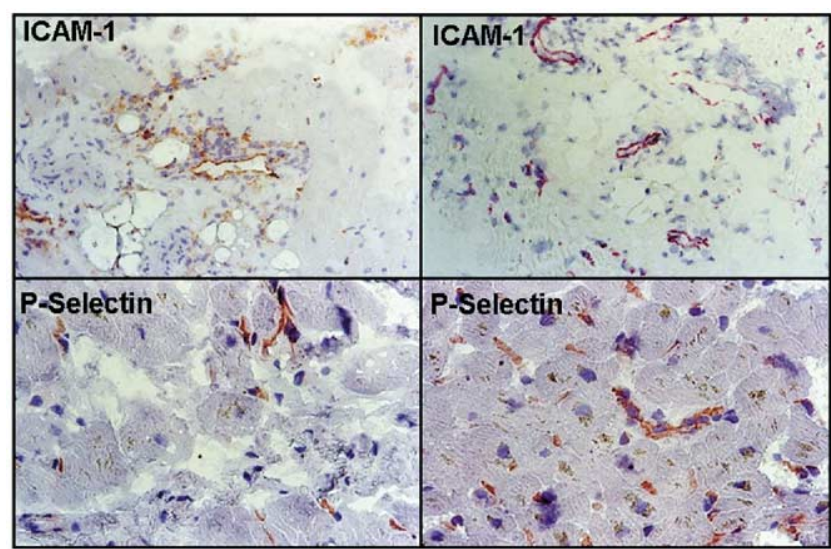

Impaired CFVR

Normal CFVR

Figure 2. Representative immunohistochemical analysis of endomyocardial biopsy specimens at 1 month after heart transplantation did not reveal differences in P-selectin and intercellular adhesion molecule 1 (ICAM-1) protein expression between patients with or without impairment of endothelium-dependent coronary flow velocity reserve (CVFR). Two biopsy samples from each time point were used. However, quantification of endomyocardial P-selectin and ICAM-1 immunoreactivity was not performed in this setting.

speculate on the source of circulating adhesion molecules after HTx. It also raises the issue of whether increased P-selectin levels, ICAM-1 levels, or both reflect endothelial cell dysfunction, platelet activation, or both.

Potential sources for circulating P-selectin are the Weibel-Palade of endothelial cells and the $\alpha$ - granules of platelets. ${ }^{10,11}$ In the present study impairment of coronary vasomotor function was associated with increased P-selectin levels, pointing to the endothelial cell as an important source. However, there is evidence that platelets might be one predominant cell fragment to release P-selectin under conditions of inflammation. Activated platelets express P-selectin on their surface, which binds to P-selectin glycoprotein ligand-1 on leukocytes and monocytes to form platelet-leukocyte aggregates. ${ }^{12}$ Indeed, activated platelets per se might not transmigrate through the endothelial layer, and platelet-leukocyte aggregate formation is one critical step in the initiation of atherothrombosis. However, activated platelets exacerbate atherosclerosis development, whereas blockade or elimination of P-selectin function reduced the atherogenesis in mice. ${ }^{13}$

ICAM-1 belongs to the immunoglobulin superfamily and is widely expressed at a basal level. It might be activated by inflammatory cytokines, including TNF- $\alpha$, in leukocytes and endothelial cells. In the present study TNF- $\alpha$ levels remained increased throughout the first year when compared with those in control subjects, and a significant transcardiac release was observed at 12 months, even under immunosuppression sufficient to prevent acute rejection. It correlated with both ICAM-1 and $\mathrm{P}$-selectin levels, suggesting that this inflammatory process is an immunologic phenomenon leading to endothelial vasomotor dysfunction.

In this regard the increased levels of soluble ICAM-1 associated with endothelial vasomotor dysfunction might point to the vessel wall as an important source during inflammation. However, in endomyocardial biopsy specimens from the allografts, no obvious differences in protein
1 month post HTx

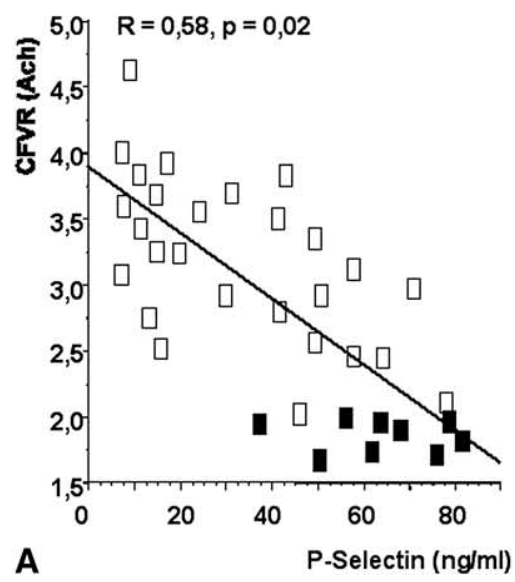

12 months post HTx

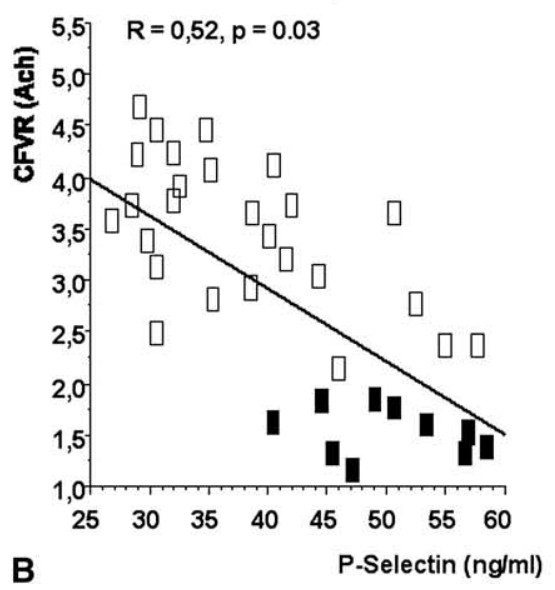

12 months post HTx

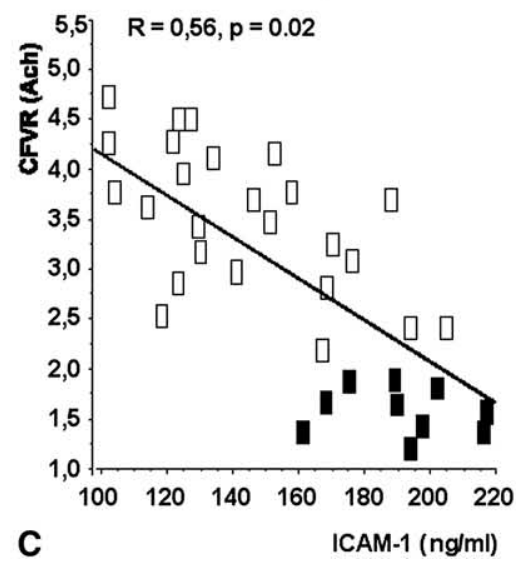

Figure 3. There was an inverse correlation between endothelium-dependent coronary flow velocity reserve (CFVR) in response to acetylcholine (Ach) and P-selectin both at 1 and 12 months after heart transplantation (HTx, A and B). In addition, CFVR after intracoronary Ach was significantly associated with higher levels of intercellular adhesion molecule 1 (ICAM-1) at 12 months after heart transplantation (C). 
expression were observed between recipients with or without impaired coronary vasomotor function. Furthermore, the cellular source of both soluble forms in cardiac allograft recipients or in patients with acute coronary syndromes is not entirely clear. One favorable hypothesis is the increased proteolytic cleavage or shedding from the cell surface, thus reflecting the increased expression of membrane-bound adhesion molecules. ${ }^{14}$ Other potential mechanisms include increased gene transcription, translational changes, or release of spliced forms. ${ }^{15}$

\section{Chronic Systemic Inflammation and Coronary Vasomotor Dysfunction}

Under homeostatic conditions, the endothelium maintains normal vascular tone and blood fluidity, and there is little to no expression of proinflammatory factors. However, various known and, to date, unknown factors, including cardiovascular disease risk factors, initiate a chronic inflammatory process that is accompanied by a loss of vasodilator and antithrombotic factors and increase vasoconstrictor and prothrombotic products. ${ }^{16}$

In the present study an enhanced expression of circulating adhesion molecules associated with coronary vasomotor dysfunction was observed in the absence of acute inflammatory processes such as infection and under immunosuppressive therapy sufficient to prevent acute rejection episodes. This suggests a chronic subtle inflammatory process triggered either by the recipient's alloimmune response, the graft itself by controlling the trafficking of effector T cells, or by other factors, such as immunosuppressive drugs and the number of established risk factors of atherogenesis. ${ }^{17-19}$

Despite the potential for therapeutic interventions, an important aspect of vasomotor dysfunction and circulation blood markers of endothelial function is their prognostic value. ${ }^{20}$ In this regard, Halcox and colleagues ${ }^{21}$ identified the pathologic coronary vasomotor response to Ach as an independent predictor of cardiovascular events, including CVD-induced death, myocardial infarction, and stroke. In addition, Hollenberg and associates ${ }^{5}$ demonstrated that heart transplant recipients with the predefined study end points of transplant vasculopathy and cardiac death showed more rapid decreases in endothelial function in serial studies.

Moreover, Ridker and coworkers ${ }^{22}$ reported that increased baseline levels of soluble adhesion molecules in apparently healthy women were associated with increasing risks for future myocardial infarction, stroke, coronary revascularization, and cardiovascular death. In addition, enhanced soluble ICAM levels were associated with poor prognosis in patients with known CAD and was an independent predictor for future cardiovascular events. ${ }^{2,23} \mathrm{Fi}$ nally, in a study by Pethig and colleagues, ${ }^{18}$ it was shown that patients with an active progressing form of transplant coronary artery disease had higher levels of highly sensitive
C-reactive protein, suggesting a systemic inflammatory state that at least reflects an ongoing atherosclerotic process.

\section{Adhesion Molecules: Therapeutic Targets in Transplant Coronary Atherothrombosis?}

The present data, together with data from previous reports, support the hypothesis that the early initiation of chronic inflammatory processes, including leukocyte rolling, tethering, attachment, and transmigration, are critical events in early atherothrombosis, as reflected by an impaired endothelial vasomotor function. ${ }^{15,19,22,24,25}$ This suggests that soluble adhesion molecules not only might be markers but also targets for therapeutic strategies. However, it remains uncertain whether long-term blockade of selectins or immunoglobulins, such as ICAM-1, would be a practical therapy under conditions of chronic inflammation. Indeed, selectins are required for effective neutrophil recruitment, and patients who lack selectins or their ligands have recurrent bacterial infections. On the other hand, partial inhibition might allow host defense to function sufficiently. In this regard it has been shown that a greater than $90 \%$ inhibition of selectins is necessary to effectively inhibit neutrophil recruitment. ${ }^{26}$ Another important aspect of selectin recruitment might be the relative organ specificity. With regard to the ischemia-reperfusion and inflammatory-type lesion, Pselectin is important for the heart and kidneys but not the lungs and liver. This highlights our incomplete understanding of the platelet-leukocyte adhesion cascade. ${ }^{27}$

\section{Summary}

The present study demonstrates an ongoing chronic subtle inflammatory process in heart transplant recipients early and 1 year after transplantation. It is reflected by persistently increased systemic P-selectin and ICAM-1 levels associated with coronary endothelial vasomotor dysfunction in the absence of acute rejection episodes or infection. It appears that the heart allograft per se is not the main source for adhesion molecule release.

Further studies are required as to why transplant recipients, even when receiving immunosuppressive therapy sufficient to prevent acute rejection episodes, express systemic and persistently high levels of circulating adhesion molecules associated with impaired endothelium-dependent coronary vasomotor function.

We thank Dr Michael Weis and Dr Sinan Pehlivanly for excellent technical assistance (catheter studies) and all the members of the Heart Transplant Team of the University of Munich for their support.

\section{References}

1. Hwang S, Ballantyne C, Sharrett A, et al. Circulating adhesion molecules VCAM-1, ICAM-1, and E-selectin in carotid atherosclerosis 
and incident coronary heart disease cases. Circulation. 1997;96: 4219-25.

2. Ridker P, Hennekens C, Roitman-Johnson B, et al. Plasma concentration of soluble intercellular adhesion molecule 1 and risks for future myocardial infarction in apparently healthy men. Lancet. 1998;129: 88-92.

3. Blankenberg S, Rupprecht H, Bickel C, et al. Circulating adhesion molecules and death in patients with coronary artery disease. Circulation. 2001;104:1336-42.

4. Valantine H. Cardiac allograft vasculopathy after heart transplantation: risk factors and management. J Heart Lung Transplant. 2004;23:18793.

5. Hollenberg S, Klein L, Parrillo J, et al. Changes in coronary endothelial function predict progression of allograft vasculopathy after heart transplantation. J Heart Lung Transplant. 2004;23:265-71.

6. Marti V, Romeo I, Aymat R, et al. Coronary endothelial dysfunction as a predictor of intimal thickening in the long term after heart transplantation. J Thorac Cardiovasc Surg. 2001;122:1174-80.

7. Koskinen P, Lemstrom K. Adhesion molecule P-selectin and vascular adhesion molecule-1 in enhanced heart allograft arteriosclerosis in the rat. Circulation. 1997;95:191-6.

8. Wildhirt S, Weis M, Schulze C, et al. Expression of endomyocardial nitric oxide synthase and coronary endothelial function in human cardiac allografts. Circulation. 2001;104suppl I:I336-43.

9. Hodgson J, Marshall J. Direct vasoconstriction and endotheliumdependent vasodilation. Mechanisms of acetylcholine effects on coronary flow reserve and arterial diameter in patients with nonstenotic coronary arteries. Circulation. 1989;79:1043-51.

10. Wagner D. The Weibel-Palade body: the storage granule for von Willebrand factor and P-selectin. Thromb Hemost. 1993;70:105-10.

11. Blann A, Nadar S, Lip G. The adhesion molecule P-selectin and cardiovascular disease. Eur Heart J. 2003;24:2166-79.

12. Huo Y, Ley K. Role of platelets in the development of atherosclerosis. Trends Cardiovasc Med. 2004;14:18-22.

13. Huo Y. Circulating activated platelets exacerbate atherosclerosis in mice deficient in apolipoprotein E. Nat Med. 2003;9:61-7.
14. Gearing A, Newman W. Circulating adhesion molecules in disease. Immunol Today. 1993;14:506-12.

15. Blankenberg S, Barbaux S, Tiret L. Adhesion Molecules and atherosclerosis. Atherosclerosis. 2003;170:191-203.

16. Widlansky M, Gokee N, Keaney J, et al. The clinical implications of endothelial dysfunction. $J$ Am Coll Cardiol. 2003;42:1149-60.

17. Chalasami G, Li Q, Komieczny B, et al. The allograft defines the type of rejection (acute versus chronic) in the face of an established effector immune response. J Immunol. 2004;172:7813-20.

18. Pethig K, Heublein B, Kutschka I, et al. Systemic inflammatory response in cardiac allograft vasculopathy. Circulation. 2000;102suppl IIII:III233-6.

19. Ross R. Atherosclerosis-an inflammatory disease. $N$ Engl J Med. 1999;340:115-26.

20. Verma S, Buchanan M, Anderson T. Endothelial function testing as a biomarker of vascular disease. Circulation. 2003;108:2054-9.

21. Halcox J, Schenke W, Zalos G. Prognostic value of coronary vascular endothelial dysfunction. Circulation. 2002;106:653-8.

22. Ridker P, Buring J, Rifai N. Soluble P-Selectin and the risk for future cardiovascular events. Circulation. 2001;103:491-5.

23. Haim M, Tanne D, Boyko V. Soluble intercellular adhesion molecule-1 and long-term risk for acute coronary events in patients with chronic coronary heart disease: data from the Bezafibrate Infarction Prevention (BIP) study. J Am Coll Cardiol. 2002;39: 1133-8.

24. Frenette P, Wagner D. Molecular medicine: adhesion molecules. N Engl J Med. 1996;334:1526-9.

25. Labarrere C, Nelson D, Miller S, et al. Value of serum-soluble intercellular adhesion molecule-1 for the non-invasive risk assessment of transplant coronary artery disease, posttransplant ischemic events and cardiac graft failure. Circulation. 2000;102:1549-55.

26. Kubes P. Therapeutic potential of inhibiting leukocyte rolling in ischemia/reperfusion. J Clin Invest. 1995;95:2510-9.

27. Ley K. The role of selectins in inflammation and disease. Trends Mol Med. 2003;9:263-8. 


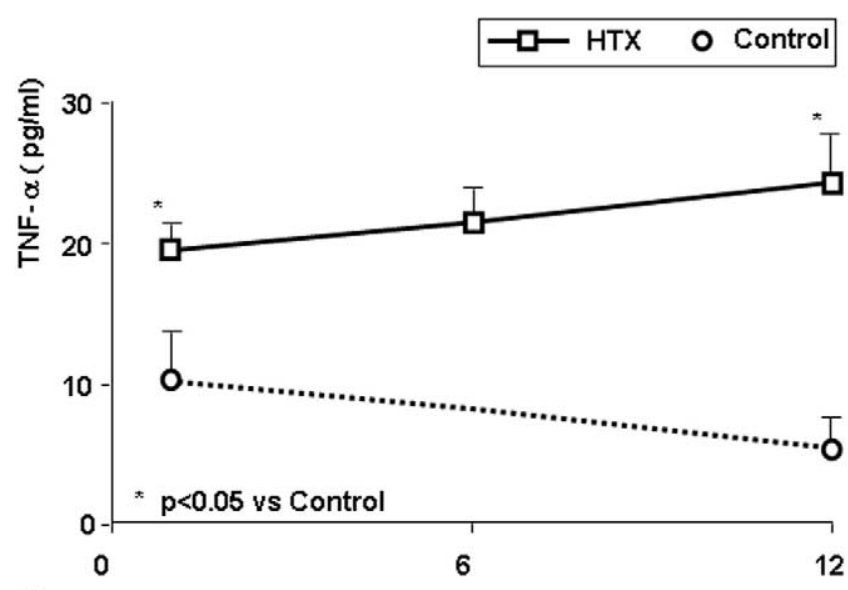

A

Months
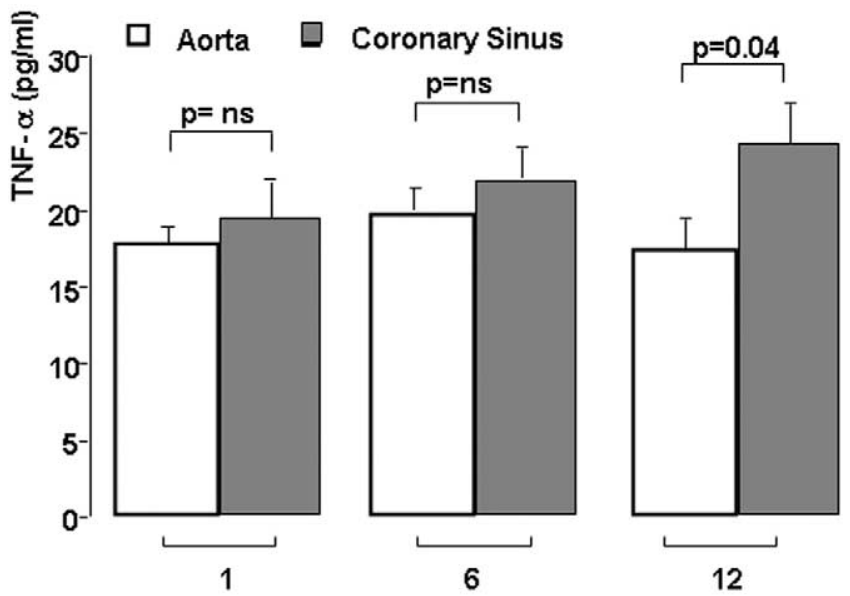

B

Months post HTx

Figure E1. Tumor necrosis factor $\alpha$ (TNF- $\alpha$ ) levels remained high throughout the first year (A). A significant transcardiac release, expressed as the difference between aortic and coronary sinus levels, was noted 1 year after heart transplantation (HTX, B).

TABLE E1. Coronary intimal thickening as detected by means of IVUS at 1 month after heart transplantation

\begin{tabular}{|c|c|c|c|c|c|c|c|}
\hline & \multicolumn{3}{|c|}{ Impaired CFVR (n = 11) } & \multicolumn{3}{|c|}{ Normal CFVR $(n=31)$} & $P$ value \\
\hline Normal morphology, n (\%) & \multicolumn{3}{|c|}{$6(54.5)$} & \multicolumn{3}{|c|}{$15(48.4)$} & NS \\
\hline \multirow{2}{*}{$\begin{array}{c}\text { Mean maximum intimal } \\
\text { thickening, } \mathbf{n}(\%)\end{array}$} & \multicolumn{3}{|c|}{ Predominant localization } & \multicolumn{3}{|c|}{ Predominant localization } & \\
\hline & Proximal & Distal & Diffuse & Proximal & Distal & Diffuse & \\
\hline Mild intimal thickening & $3(27.3)$ & 0 & 0 & $11(35.4)$ & 0 & 0 & NS \\
\hline Moderate intimal thickening & 0 & 0 & $1(9.1)$ & $1(3.2)$ & 0 & $2(6.5)$ & NS \\
\hline Severe intimal thickening & 0 & 0 & $1(9.1)$ & 0 & 0 & $2(6.5)$ & NS \\
\hline
\end{tabular}



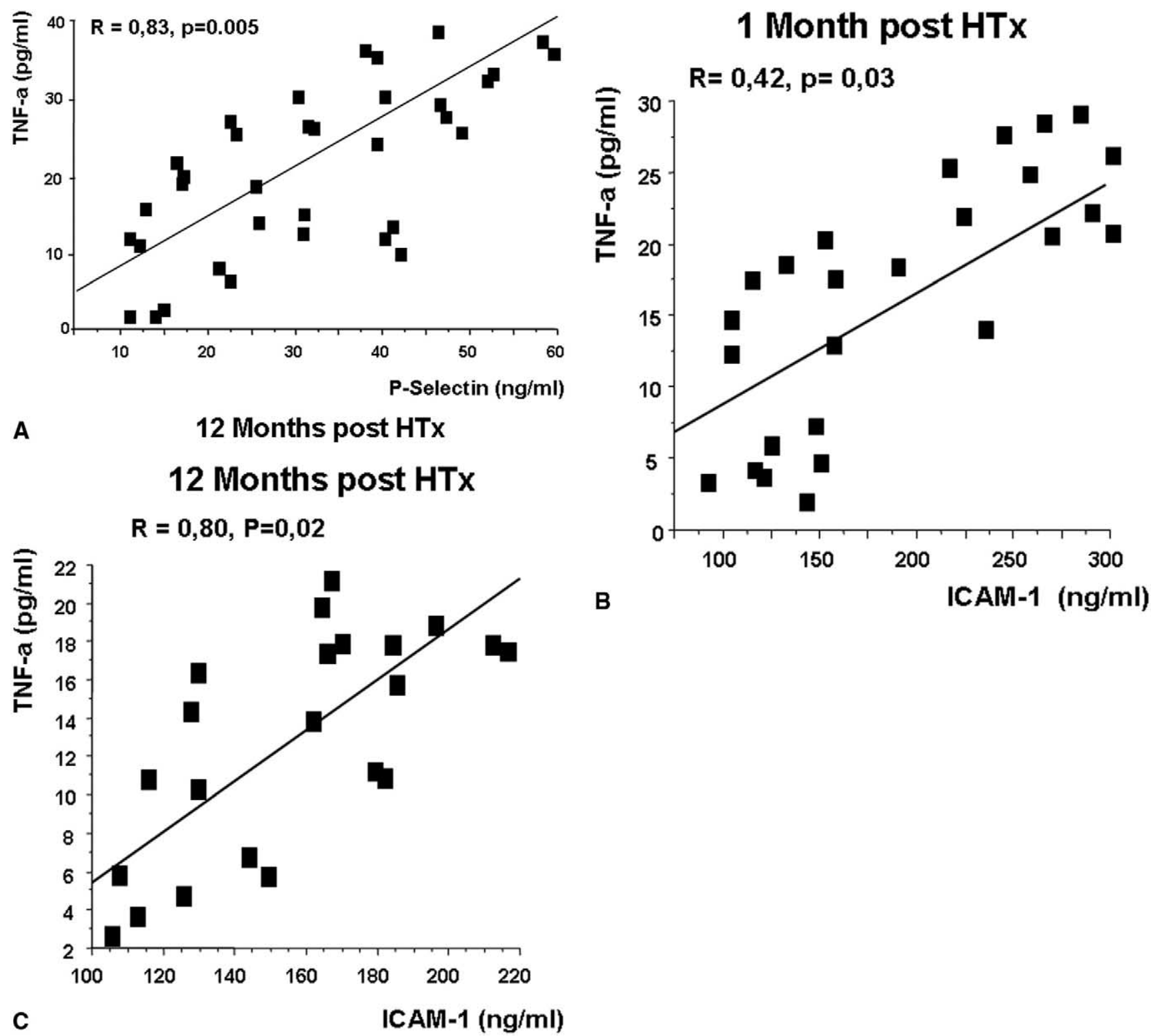

C

ICAM-1 (ng/ml)

Figure E2. A close and significant correlation between the proinflammatory cytokine tumor necrosis factor $\alpha$ (TNF- $\alpha$ ) and P-selectin at 1 year after heart transplantation (HTX) is shown (A). In addition, there was a significant inverse correlation between TNF- $\alpha$ and intercellular adhesion molecule 1 (ICAM-1) both at 1 month and 1 year after heart transplantation (B and C). 